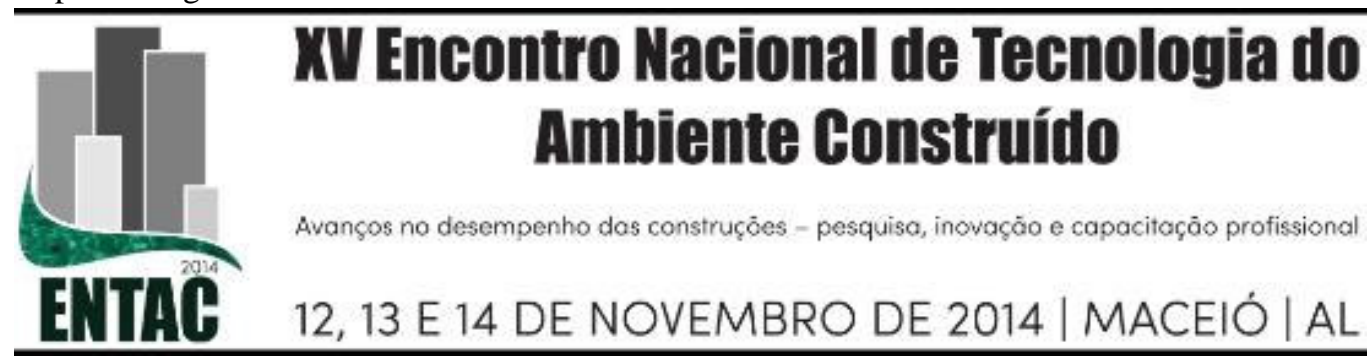

\title{
ESTUDO EXPERIMENTAL COM SISTEMAS PASSIVOS DE RESFRIAMENTO EM FLORIANÓPOLIS
}

\author{
GONZÁLEZ CRUZ, Eduardo (1); ORDENES MIZGIER, Martín (2); RUTTKAY \\ PEREIRA, Fernando (3); GÜTHS, Saulo (4)
}

(1)UFSC, edugoncruz@gmail.com, (2)UFSC, martin@arq.ufsc.br, (3) UFSC, feco@ arq.ufsc.br, (4)

UFSC, saulo@lmpt.ufsc.br

\begin{abstract}
RESUMO
O trabalho de pesquisa apresentado tem como objetivo avaliar o comportamento térmico de cinco sistemas passivos de resfriamento (SPR), que utilizam como base uma configuração de teto-reservatório onde são aplicados os princípios de inércia térmica, resfriamento evaporativo indireto e resfriamento radiativo noturno. Para isto foi desenvolvido um trabalho experimental baseado na metodologia proposta por González (2013). A pesquisa analisa as temperaturas características, fatores decrementais e potenciais de resfriamento nas diferentes estratégias dos sistemas estudados. As correlações obtidas permitem comparar e prever o comportamento térmico dos sistemas em função das variações climáticas em Florianópolis. Os resultados preliminares permitem concluir que: os sistemas avaliados apresentam um potencial de resfriamento médio (PRM) que varia entre $119 \mathrm{~W} / \mathrm{m}^{2}$-dia e $255 \mathrm{~W} / \mathrm{m}^{2}$-dia, com valores máximos de até $319 \mathrm{~W} / \mathrm{m}^{2}$-dia; estas técnicas de resfriamento passivo podem reduzir a temperatura máxima interna em até $10,4{ }^{\circ} \mathrm{C}$ se comparado com a temperatura máxima externa; é possível reduzir a amplitude da temperatura interna em função da amplitude externa (fator decremental médio entre 0,34 e 0,70). Estes resultados confirmam a viabilidade de aplicação das técnicas de resfriamento passivo para o condicionamento térmico de edificações no período de verão em Florianópolis.
\end{abstract}

Palavras-chave: resfriamento passivo, resfriamento evaporativo indireto, resfriamento radiativo noturno, inércia térmica, estudo experimental, clima quente e úmido, arquitetura bioclimática.

\begin{abstract}
The research work presented in this publication aims to evaluate the thermal behavior on five different passive cooling systems (PCS). All systems have a common configuration of roof pond with applied strategies of thermal mass, indirect evaporative cooling, and night radiative cooling. The experimental arrangement follows the methodology described by González (2013). The research method analyses characteristic temperatures, decremental factor and cooling potential in the different system strategies adopted. Correlation between this variables and weather data from Florianópolis allows comparing and predicting the thermal behavior of these systems. Main results have shown that: (i) passive cooling systems can achieve an average cooling potential of 119 255 W/m ${ }^{2}$-day, with a maximum value of 319 $\mathrm{W} / \mathrm{m}^{2}$-day; (ii) these passive cooling techniques can reduce the maximum indoor temperature in $10.4{ }^{\circ} \mathrm{C}$ if compared to the maximum outdoor temperature; (iii) it is possible to reduce, in average, on $34 \% \sim 70 \%$ the indoor temperature amplitude compared to the outdoor amplitude. These results confirm the effective application of passive cooling techniques for natural thermal conditioning on buildings during the summer conditions of Florianópolis.
\end{abstract}

Keywords: passive cooling, indirect evaporative cooling, nocturnal radiative cooling, thermal inertia, experimental study, warm and humid climate, bioclimatic architecture. 


\section{INTRODUÇÃ̃O}

De acordo com dados do Balanço Energético Nacional (BEN, 2013), as edificações são responsáveis por $48 \%$ de toda a energia elétrica utilizada no Brasil. Tanto em edifícios residenciais (24\%) como comerciais (16\%) e públicos $(8 \%)$, este consumo pode ser explicado pela necessidade de sistemas de climatização que facilitam o desenvolvimento das atividades humanas dentro dos espaços construídos.

No entanto, as condições térmicas internas podem ser condicionadas através de estratégias de resfriamento passivo evitando assim o consumo energético associado aos sistemas artificiais de climatização e suas consequências no impacto ambiental.

Entre as estratégias de climatização passiva, o uso de um teto-reservatório como sistema de resfriamento (evaporativo indireto ou radiativo) vem sendo estudado em locais com climas secos (GIVONI, 1981, 1994; TANG, 2003; KHARRUFA, 2008), e em climas quentes e úmidos tem sido demonstrada a real capacidade desta tecnologia para reduzir a temperatura interna com relação à temperatura externa (SÁNCHEZ, 1993; GIVONI, 2011; GONZÁLEZ, 2013).

O conforto térmico exige uma faixa reduzida de variação para a temperatura de ar interna. As edificações com maior capacidade de armazenamento térmico tendem a apresentar oscilações de temperaturas reduzidas, menores à amplitude de temperatura externa. As vantagens da inércia térmica para atingir condições de conforto em climas tropicais úmidos e semi-úmidos, vêm sendo reivindicada por diversos autores nas últimas décadas (SZOKOLAY, 2000; DORNELLES, 2004). Especificamente, para o clima de Florianópolis, PAPTS (1999) indica que "... o uso de inércia térmica tem um bom desempenho térmico tanto no período quente quanto no período frio...".

Neste contexto, o trabalho de pesquisa apresentado tem como objetivo avaliar o comportamento térmico de cinco sistemas passivos de resfriamento (SPR), que utilizam como base uma nova configuração de teto-reservatório (GONZÁLEZ, 2013) onde são aplicados os princípios de inércia térmica, resfriamento evaporativo indireto e resfriamento radiativo noturno.

\section{METODOLOGIA}

\subsection{Florianópolis: Seu clima de verão e as condições de conforto.}

A cidade de Floriaópolis encontra-se na região sul do país na latitude $27,67^{\circ} \mathrm{Sul}$ e longitude $48,34^{\circ}$ Oeste. Seu clima, temperado subtropical -Cfa- de acordo com a classificação de Köppen-Geiger, está caracterizado por uma temperatura média anual de $20,7^{\circ} \mathrm{C}$, com uma variação entre $10,6^{\circ} \mathrm{C}$ e $28,2^{\circ} \mathrm{C}$. Sua amplitude térmica média diária é de $7,2^{\circ} \mathrm{C}$, com valores entre $2{ }^{\circ} \mathrm{C}$ e $16^{\circ} \mathrm{C}$. As frentes frias provenientes do sul e a presença de chuvas distribuídas ao longo do ano favorecem uma alta variabilidade de temperaturas em breves períodos de tempo. A umidade relativa média anual é de $82,7 \%$ e a precipitação total é de $1.600 \mathrm{~mm}$ anuais. Os meses de maior precipitação coincidem com os meses mais quentes, entre setembro e março. A maior nebulosidade acontece nos meses de verão, em que supera os 6,2/10 de nebulosidade média anual. $O$ vento Norte é predominante ao longo do ano, seguido pelo vento Sul e Nordeste. A média diária de radiação global horizontal varia entre $2,7 \mathrm{kWh} / \mathrm{m}^{2}$ em julho e $6,1 \mathrm{kWh} / \mathrm{m}^{2}$ em

Janeiro. Estas informações climáticas foram obtidas do arquivo BRA_Florianopolis.838990_SWERA.epw (www.labeee.ufsc.br). 
Durante o período de verão a média das temperaturas máximas é de $27,9{ }^{\circ} \mathrm{C}$, com valores picos de até $34^{\circ} \mathrm{C}$. A temperatura média é de $24,1^{\circ} \mathrm{C}$, com uma variação entre $20,1^{\circ} \mathrm{C}$ e $28,2^{\circ} \mathrm{C}$. No entanto, durante o período de ensaio os registros de temperatura superaram os dados estatísticos. Isto pode ser observado na Figura 1 em que são apresentados os valores máximos (Tmax) e mínimos (Tmin) diários (arquivo climático .epw e registrados durante o estudo) e a zona de conforto correspondente calculada a partir da norma ASHRAE Standard 55 para edificações com ventilação natural.

A análise bioclimática do arquivo TRY para Florianópolis para o período de verão entre os dias 15 de novembro e 31 de março indica um percentual de $83,3 \%$ de desconforto para este período. Deste percentual, $73,4 \%$ corresponde a uma situação de desconforto por calor.

Figura 1 - Temperaturas máximas e mínimas em Florianópolis e em ensaios.

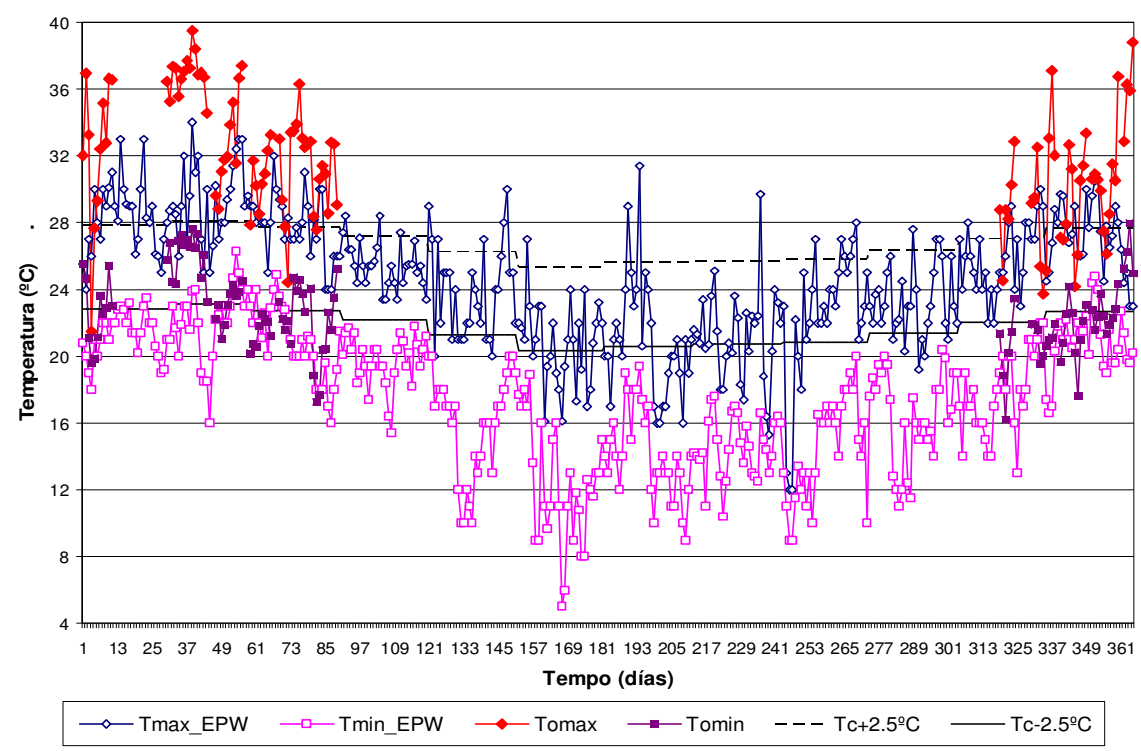

Fonte: próprio autor (2014)

\subsection{Descrição do dispositivo experimental}

A bancada experimental conta com dois módulos com dimensões idênticas, fabricados com lâminas de madeira compensada de $18 \mathrm{~mm}$ de espessura e pintados na cara exterior com tinta esmaltada na cor branca (Figuras 3 e 4). Os módulos, experimental (ME) e de controle (MC) foram revestidos internamente nas suas paredes e pisos com camadas de $45 \mathrm{~mm}$ de poliestireno expandido. A diferença entre os módulos encontra-se apenas na cobertura. Enquanto o MC (Figura 3A) apresenta uma cobertura com alto isolamento $\left(\mathrm{U}=0,24 \mathrm{~W} / \mathrm{m}^{2} \mathrm{~K}\right.$ formado por uma camada de $18 \mathrm{~mm}$ de cobertura em madeira compensada), o ME considera a instalação de um reservatório metálico de água, fabricado com uma lâmina galvanizada de 1,2 $\mathrm{mm}$ de espessura e pintado na cor branca. Nas Figuras 3B e 4B é possível observar os detalhes e especificações para a seção da cobertura. $\mathrm{O}$ fechamento do ME, sobre o reservatório de água, e os diferentes níveis de água adotados variam em função dos diferentes experimentos realizados. Isto é explicado em detalhe na seguinte seção. 


\section{Figura 2 - Módulo de Controle (A) e Experimental (B) utilizados na pesquisa.}

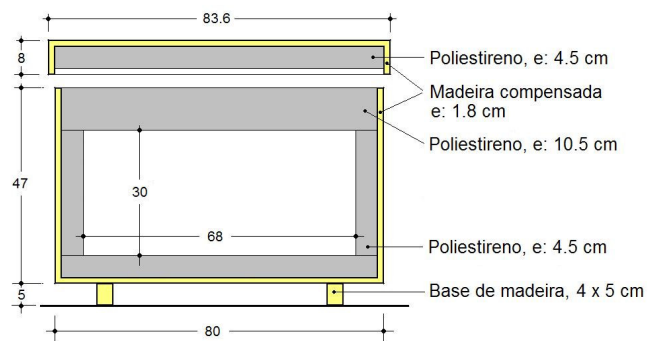

(A)

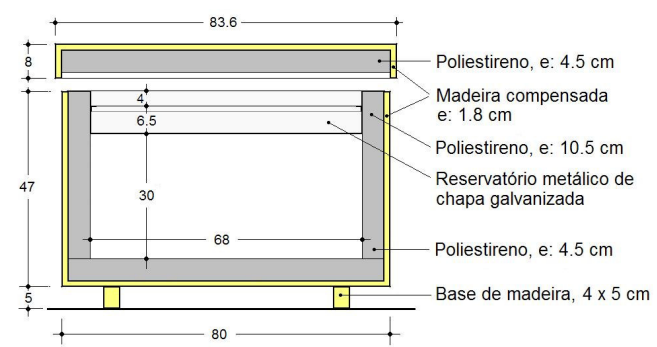

(B)

Fonte: próprio autor (2014)

Figura 3 - Bancada experimental - MC e ME (A), ME e reservatório de água (B)

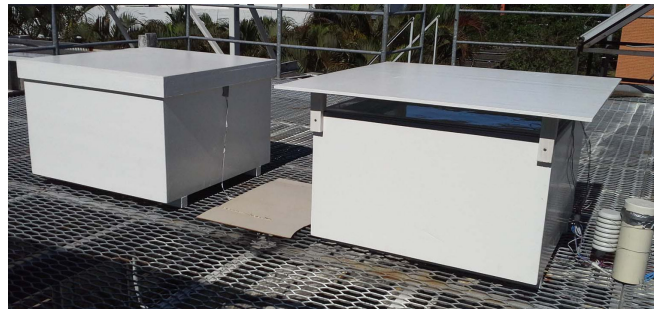

(A)

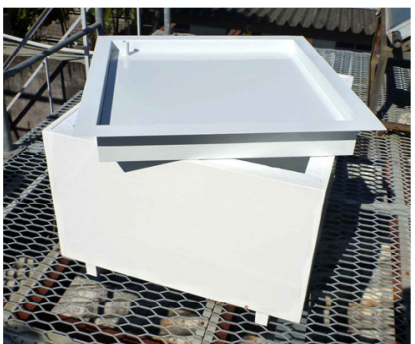

(B)

Fonte: próprio autor (2014)

\subsection{Descrição do sistema de aquisição de dados}

Nestes experimentos foram monitorados e registrados dados de temperatura, umidade relativa, fluxo de calor com intervalos de um minuto e a quantidade de água evaporada a cada dois dias. O sistema de aquisição de dados é composto por uma Unidade de aquisição e registro de dados Agilent-HP, modelo 34970A, resolução $0,1 \mu \mathrm{V}$, conectado a um computador com o software Agilent BenchLink Data Logger para aquisição de dados. A temperatura do ar (dentro e fora dos módulos), e a temperatura da água foram medidas com termopares (7) tipo T (cobre-constantan). Foi adotada uma junção de referência em que a temperatura é medida com um termistor NTC de $30 \mathrm{KOhm}$, incerteza de $0,15{ }^{\circ} \mathrm{C}$, com intervalo de confiança de $95 \%$. Para a medição do fluxo de calor foram utilizados dois transdutores de fluxo de calor de tipo gradiente tangencial. (GÜTHS, 1998). Todos os sensores foram calibrados antes da sua utilização na bancada experimental. A umidade relativa do ar foi medida com um higrômetro tipo capacitivo, marca Honneywel, com uma faixa de medição de 5 a $98 \%$ e uma incerteza de $3 \%$.

\subsection{Descrição dos sistemas avaliados.}

O módulo experimental (ME) apresenta uma configuração diferente para cada um dos ensaios realizados em função do Sistema Passivo de Resfriamento (SPR) a avaliar. Na Figura 5 é descrita a configuração do ME em cada SPR, assim como o objetivo de cada ensaio e a forma de operação nos períodos diurno e noturno. 
O período de monitoramento dos sistemas foi iniciado no dia 15 de novembro de 2013 e foi encerrado no dia 07 de março de 2014. O tempo de monitoramento de cada ensaio foi de 15 dias para o SPR 1 (Experimento 1), 15 dias para o SPR 2 (Experimento 5), 10 dias para o SPR 3 (Experimento 6), 14 dias para o SPR 4 (Experimento 4) e 10 dias para o SPR 5 (Experimento 7).

Figura 4 - Sistemas avaliados, descrição e condições de operação.

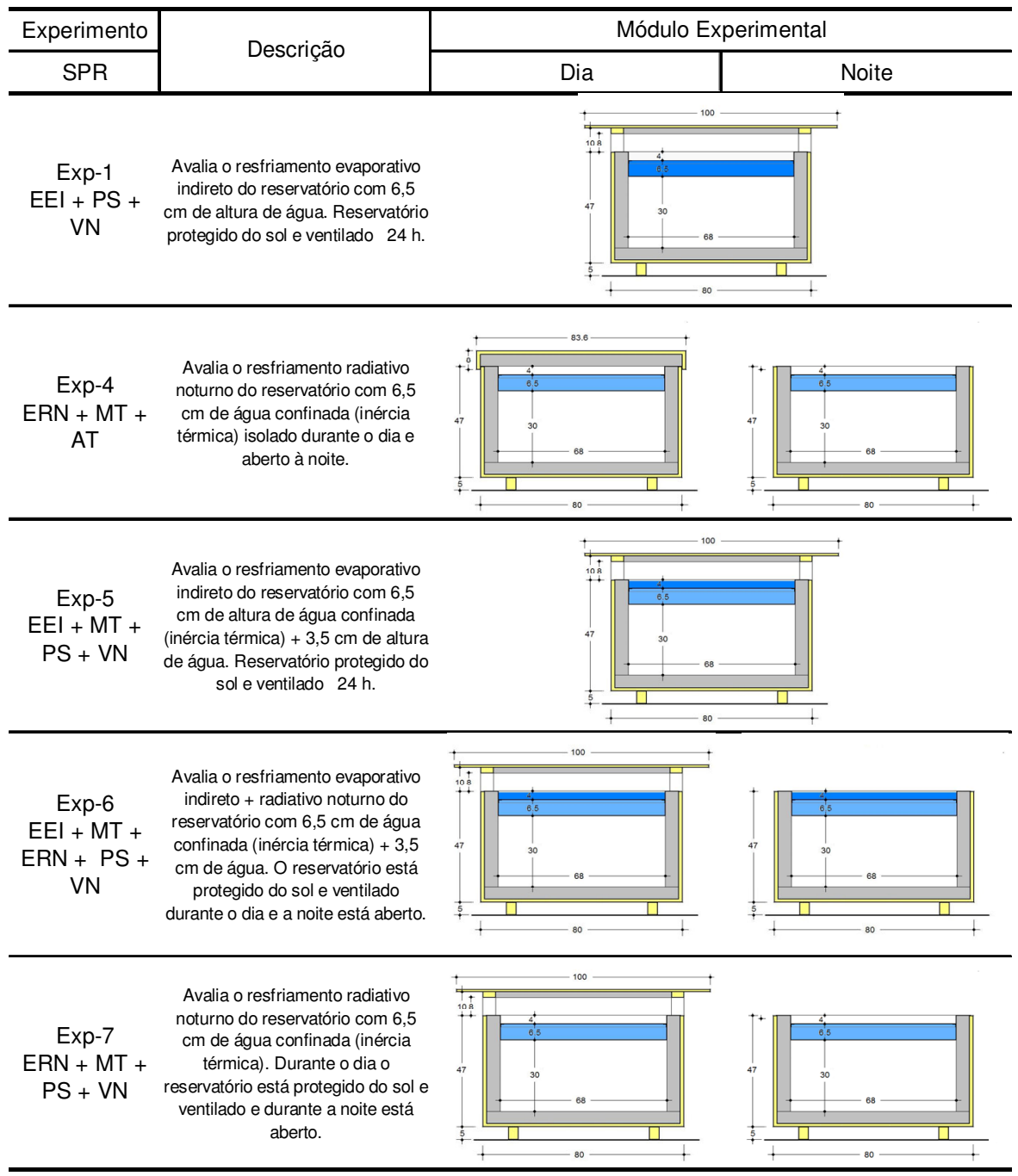

EEI=resfriamento evaporativo indireto; $\mathrm{PS}=$ proteção solar; $\mathrm{VN}=$ ventilação natural; $\mathrm{MT}=$ =massa térmica; $\mathrm{ERN}=$ resfriamento radiativo noturno; $\mathrm{AT}=$ isolamento térmico.

Fonte: próprio autor (2014)

\section{ANÁLISE DOS RESULTADOS}

\subsection{Temperaturas características}

A análise comparativa das temperaturas características (temperatura máxima -Tmax-, temperatura mínima -Tmin-, temperatura média -Tmed- e amplitude de temperatura DT-) no módulo experimental com os valores correspondentes ao módulo de controle e o ambiente externo permitem caracterizar o comportamento térmico dos SPR. A 
eficiência de resfriamento será maior na medida em que consiga reduzir as temperaturas máximas, mínimas e médias em relação às temperaturas no módulo de controle. Por outro lado, o sistema pode apresentar um melhor comportamento térmico quanto menor seja o fator decremental e maior seja a diferença entre as temperaturas médias internas do MC e do ME.

Na Figura 5 são apresentadas as temperaturas máximas, médias e mínimas do ambiente e do ME para os 5 experimentos. Observa-se primeiramente a importante redução de Temax no ME em comparação com a Tomax do ambiente externo. Esta redução atinge $10,4{ }^{\circ} \mathrm{C}$ no experimento 1 . Nos experimentos 4,5 e 6 estas diferenças oscilam entre 8,8 ${ }^{\circ} \mathrm{C}$ e $10^{\circ} \mathrm{C}$. A maior diferença média entre as máximas foi registrada no experimento 5 $\left(7,7^{\circ} \mathrm{C}\right)$, em que as condições climáticas foram mais estáveis e registraram as maiores temperaturas máximas. É importante destacar que a Temax no ME é, em média, inferior à temperatura média do ambiente externo em 0,3 ${ }^{\circ} \mathrm{C}\left(\right.$ Exp-1), em $1,4{ }^{\circ} \mathrm{C}($ Exp-5) e em 0,8 ${ }^{\circ} \mathrm{C}$ (Exp-6). No Exp-4 as duas temperaturas são, em média, iguais. No caso do Exp7, Temax é $1,7^{\circ} \mathrm{C}$ superior, em média, se comparada com a temperatura média exterior. Ao comparar as temperaturas mínimas observa-se que o $\mathrm{ME}$ alcança uma Temin média 0,4 ${ }^{\circ} \mathrm{C}$ inferior ao $\mathrm{MC}$ no Exp-1, 0, $1^{\circ} \mathrm{C}$ inferior no Exp-4 e Exp-7 e 0,6 inferior no Exp6. Para o Exp-5 a Temin é superior em $0,1^{\circ} \mathrm{C}$ à temperatura mínima do $\mathrm{MC}$.

Figura 5 - Temperaturas Max, Min y Med do ambiente externo e do ME.

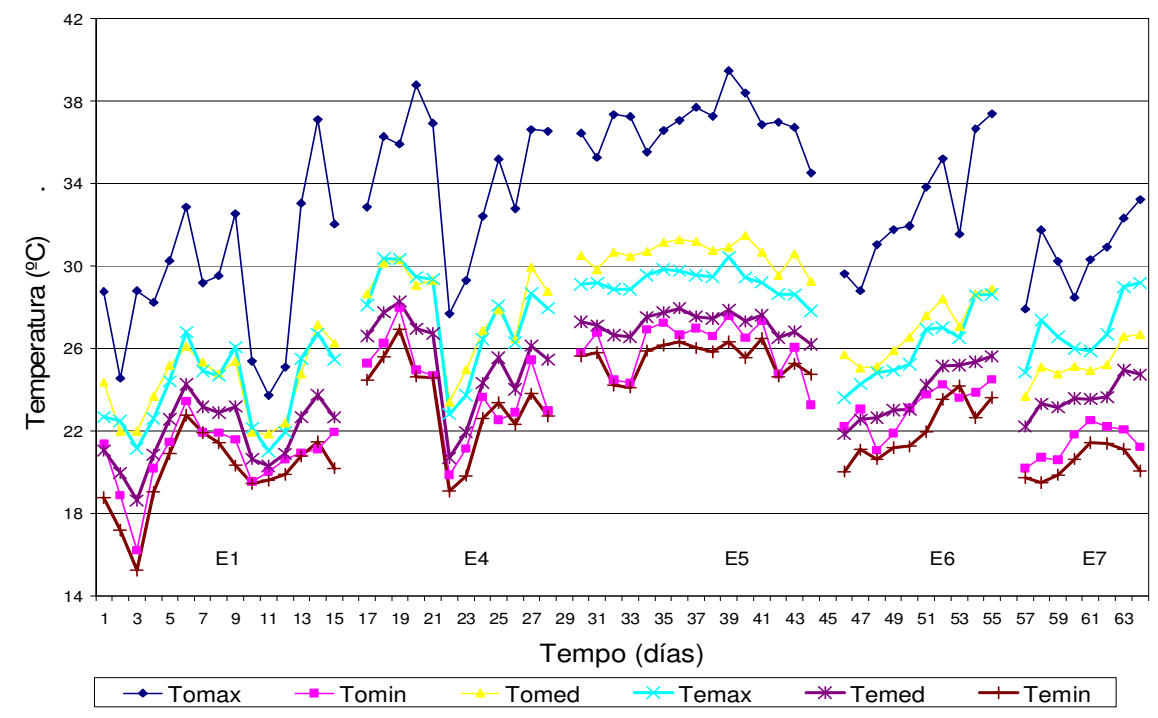

Fonte: próprio autor (2014)

Dessa forma, é possível obter as seguintes conclusões: os cinco sistemas avaliados apresentam uma importante capacidade para reduzir a temperatura máxima interna; em todos os experimentos foram obtidas temperaturas mínimas inferiores (ou muito próximas) às mínimas do ambiente externo e no $\mathrm{MC}$; e como consequência do anterior, observa-se uma redução da temperatura média do $\mathrm{ME}$ em função do $\mathrm{MC}$ e uma redução na oscilação da temperatura interna em função à temperatura externa e à do $\mathrm{MC}$. $\mathrm{Na}$ Tabela 1 são apresentadas as temperaturas médias do ambiente externo, MC e ME de cada experimento junto com os fatores decrementais de cada caso. Em termos absolutos, é possível observar que é no Exp-5 onde é obtida a maior diferença média entre Tc e Te $\left(3,2^{\circ} \mathrm{C}\right)$ com um valor máximo de $3,9^{\circ} \mathrm{C}$. Em segundo lugar, encontra-se o Exp-6 com uma diferença média de $3,0^{\circ} \mathrm{C}$ (máxima de $4,0{ }^{\circ} \mathrm{C}$ ), em terceiro lugar o Exp-4 com 2,6 
${ }^{\circ} \mathrm{C}$ (máxima de $3,7{ }^{\circ} \mathrm{C}$ ), em quarto lugar o Exp-1 com $2,3{ }^{\circ} \mathrm{C}$ (máxima de $3,6{ }^{\circ} \mathrm{C}$ ) e em último lugar o Exp-7 $\operatorname{com} 1,5^{\circ} \mathrm{C}$ de diferença média e um valor máximo de $2,0^{\circ} \mathrm{C}$.

Assim, é possível comprovar a influência da inércia térmica (representada pela água do reservatório) para reduzir a amplitude de temperatura interna do $\mathrm{ME}$ em relação à temperatura do ambiente externo e a do MC. Na Tabela 1 são apresentados os fatores decrementais (FD) em função do ao ambiente externo e ao MC. Observa-se que os casos com menor FD (Exp-5 FD=0,34 e Exp-6 FD=0,42) correspondem aos SPR com maior quantidade de água no reservatório $(29,5$ lts de água confinada $+15,9$ lts de água exposta $=45,4$ lts). Nos experimentos 1,4 e 7 a quantidade de água no reservatório é de 29,5 lts. O FD nestes sistemas evidencia a capacidade de cada um deles para manter a temperatura da água com a menor oscilação. Assim, o Exp-4 e o Exp-1 apresentam o terceiro e quarto menor valor para o FD. O Exp-7 apresenta o maior valor para o FD dentre os casos estudados.

\section{Tabela 1 - Temperaturas características e fatores decrementais}

\begin{tabular}{|c|c|c|c|c|c|c|c|c|c|c|c|c|}
\hline \multirow{2}{*}{ Experimento / SPR } & \multicolumn{2}{|c|}{ Temp Ext } & \multicolumn{2}{|c|}{ Tem MC } & \multicolumn{2}{|c|}{ Temp ME } & \multicolumn{3}{|c|}{$\mathrm{Tc}-\mathrm{Te}$} & Agua & \multicolumn{2}{|c|}{ Factor Decremantal } \\
\hline & Tom & $\Delta \mathbf{T}$ & Tcm & DvEst & Tem & DvEst & $\stackrel{\circ}{\circ}$ & DvEst & $\max$ & Lts & $\Delta \mathrm{Te} / \Delta \mathrm{To}$ & $\Delta \mathrm{Te} / \Delta \mathrm{Tc}$ \\
\hline $1 / \mathrm{EE}+\mathrm{PS}+\mathrm{VN}$ & 24.2 & 8.7 & 24.1 & 1.8 & 21.8 & 1.6 & 2.3 & 0.8 & 3.6 & 29.5 & 0.47 & 0.43 \\
\hline 4 / ERN+AT & 28.0 & 10.3 & 28.0 & 2.2 & 25.4 & 2.3 & 2.6 & 0.5 & 3.7 & 29.5 & 0.43 & 0.39 \\
\hline $5 / \mathrm{EEI}+\mathrm{MT}+\mathrm{PS}+\mathrm{VN}$ & 30.6 & 10.8 & 30.4 & 0.6 & 27.2 & 0.5 & 3.2 & 0.4 & 3.9 & 45.4 & 0.34 & 0.30 \\
\hline $6 / \mathrm{EEI}+\mathrm{ERN}+\mathrm{MT}+\mathrm{PS}+\mathrm{VN}$ & 26.9 & 9.7 & 26.8 & 1.5 & 23.9 & 1.4 & 3.0 & 0.7 & 4.0 & 45.4 & 0.42 & 0.37 \\
\hline $7 /$ ERN $+P S+V N$ & 25.2 & 9.2 & 25.1 & 1.1 & 23.6 & 0.9 & 1.5 & 0.4 & 2.0 & 29.5 & 0.70 & 0.59 \\
\hline
\end{tabular}

Fonte: próprio autor (2014)

Figura 6 - Correlação entre Tic (MC) e Tie (ME).

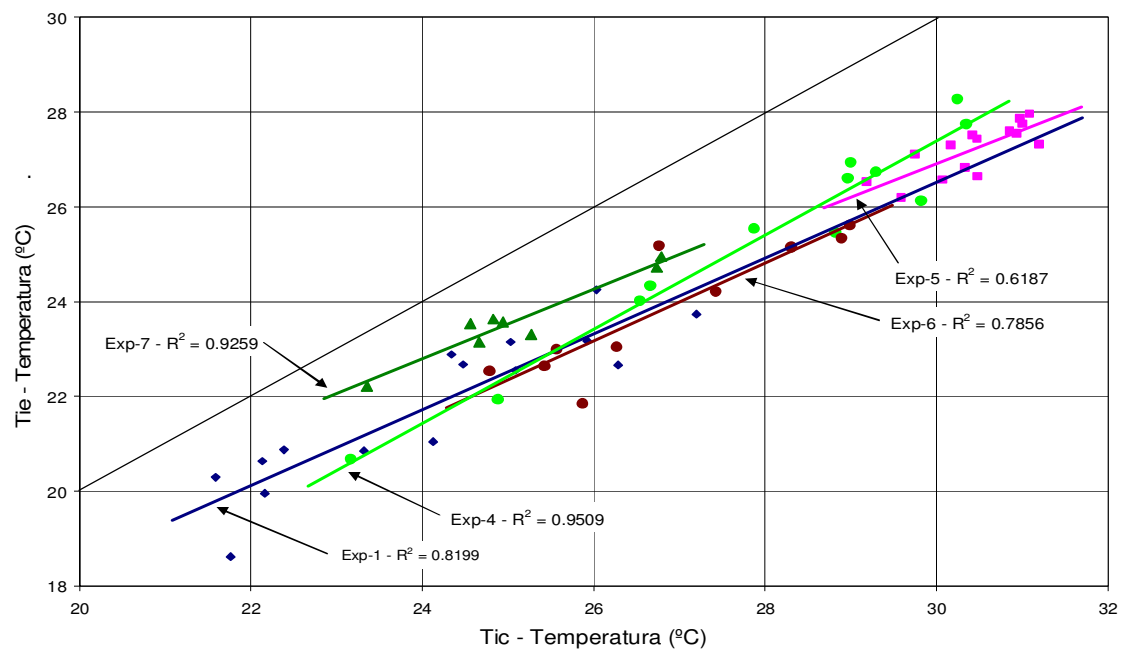

Fonte: próprio autor (2014)

Na Figura 6 são apresentados os valores de correlação entre as temperaturas médias do MC e do ME para cada experimento. Observa-se que em todos os SPR o ME registrou temperaturas médias inferiores ao MC. No gráfico foram plotadas as linhas de tendência para cada conjunto de dados e os coeficientes de correlação para cada caso. Foram obtidos valores altos para os coeficientes de correlação entre as duas variáveis, sendo maiores nos casos com RRN, e menores onde o EEI está presente. 
Nos experimentos 1, 5, 6 e 7 a análise de regressão linear indica que a diferença entre Tic e Tie tende a aumentar na medida em que aumenta a temperatura média do MC. Isto mostra que a eficiência de resfriamento aumenta na medida em que aumenta a temperatura no MC. No entanto, a linha de tendência do experimento 4 (ERN+AT) apresenta uma situação diferente. Neste caso, a diferença entre Tic e Tie se mantem constante independentemente do valor de Tic. Considerando que existe uma correlação muito alta entre a temperatura do ambiente externo e a do MC, é possível afirmar que a eficiência de resfriamento nestes sistemas será maior em dias mais quentes.

Também na Figura 6, ao comparar a eficiência dos SPR para temperaturas entre $26^{\circ} \mathrm{C}$ e $30{ }^{\circ} \mathrm{C}$ (onde existe maior necessidade de resfriamento) é possível observar que os sistemas em que se consegue a maior diferença de temperatura correspondem, de forma decrescente, aos experimentos $6,1,5,4$ y 7 respectivamente.

\subsection{Análise do Potencial de Resfriamento Médio (PRM)}

O Potencial de Resfriamento Médio (PRM) é definido como "a quantidade de energia por unidade de tempo e de superfície que pode ser retirada pelo sistema como valor médio durante um período de 24 horas" (González e González, 2013). Este potencial é calculado como função da diferença entre as temperaturas médias dos módulos de controle e experimental, como mostra a equação 1 .

$$
P R M=\frac{C P C \times(T c m-T e m) \times 24 h}{A}
$$

Onde, $\mathrm{CPC}=$ Coeficiente de Perda de Calor do módulo $(1,5 \mathrm{~W} / \mathrm{K})$;

$\mathrm{Tcm}=$ temperatura média do módulo de controle em $24 \mathrm{~h}\left({ }^{\circ} \mathrm{C}\right)$;

Tem = temperatura media do módulo experimental em $24 \mathrm{~h}\left({ }^{\circ} \mathrm{C}\right)$.

A = área da superfície efetiva do reservatório $\left(0,4543 \mathrm{~m}^{2}\right)$

Os módulos utilizados neste trabalho de pesquisa tem características construtivas e dimensionais idênticas às usadas por González e González (2013). Dessa forma, é possível adotar o mesmo coeficiente de perda de calor $(\mathrm{CPC}=1,5 \mathrm{~W} / \mathrm{K})$ no caso atual. A superfície do reservatório no teto do módulo experimental em contato com a superfície interior tem uma área efetiva de resfriamento de $\mathrm{A}=0,4543 \mathrm{~m}^{2}$.

Tabela 3 - Estimativa do Potencial de Resfriamento Médio (PRM)

\begin{tabular}{c|c|c|c|c|c|c|c}
\hline \multicolumn{2}{c|}{ CPC $=1.5 \mathrm{~W} / \mathrm{K}$} & \multicolumn{5}{c}{ Superfície de cobertura $(\mathrm{A})=0,4543 \mathrm{~m}^{2}$} & \multicolumn{2}{c}{ PRMmax } \\
\hline Experimento & Tc & Te & DT & Wh $/$ dia & Wh $/ \mathrm{m}^{2}$ dia & Desvest & Wh $/ \mathrm{m}^{2}$ dia \\
\hline 1 & 24.12 & 21.83 & 2.30 & 82.7 & $\mathbf{1 8 2 . 1}$ & 61.4 & $\mathbf{2 8 6 . 9}$ \\
\hline 4 & 27.97 & 25.37 & 2.60 & 93.6 & $\mathbf{2 0 6 . 0}$ & 40.1 & $\mathbf{2 9 2 . 8}$ \\
\hline 5 & 30.43 & 27.21 & 3.22 & 115.9 & $\mathbf{2 5 5 . 2}$ & 29.8 & $\mathbf{3 0 7 . 0}$ \\
\hline 6 & 26.83 & 23.86 & 2.97 & 107.0 & $\mathbf{2 3 5 . 5}$ & 55.5 & $\mathbf{3 1 8 . 9}$ \\
\hline 7 & 25.14 & 23.64 & 1.51 & 54.2 & $\mathbf{1 1 9 . 4}$ & 62.9 & $\mathbf{1 5 8 . 9}$ \\
\hline
\end{tabular}

Fonte: próprio autor (2014)

A Tabela 3 apresenta os resultados de cálculo do PRM para cada experimento. O resultado destes cálculos permite estabelecer uma hierarquia entre os sistemas estudados. É importante destacar que, tirando o Exp-7, foram registrados PRMmax entre 287 e $319 \mathrm{Wh} / \mathrm{m}^{2}$-dia. Considerando que os experimentos foram realizados em diferentes condições climáticas, não é possível realizar uma comparação dos valores 
médios de PRM porque a eficiência de resfriamento deles aumenta ou diminui com as condições climáticas locais. É por este motivo que foi utilizado a análise de regressão linear para comparar a tendência dos cinco experimentos.

\section{Figura 7 - Correlação entre a depressão de TBU (TBS-TBU) e o PRM}

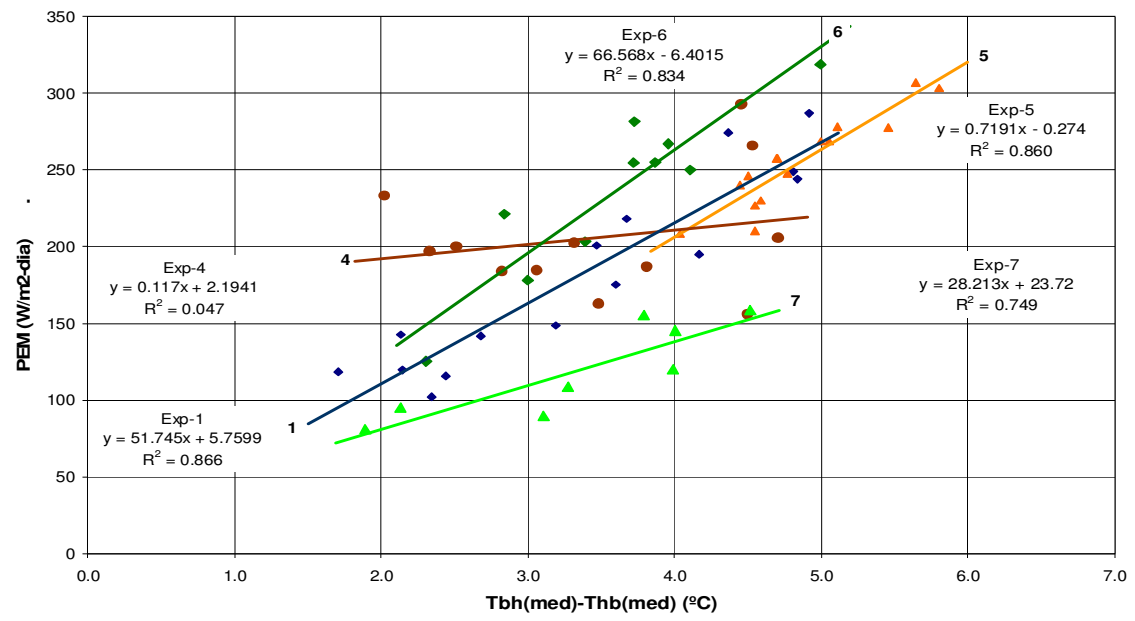

Fonte: próprio autor (2014)

A Figura 7 apresenta a correlação entre os dados de depressão de temperatura de bulbo úmido (TBS-TBU) e a diferença de temperatura média entre o $\mathrm{MC}$ e o $\mathrm{ME}$ (Tcm-Tem) de cada experimento. É possível observar que os sistemas que utilizam o EEI (Exp-1, 5 e 6) apresentam retas com uma inclinação muito semelhante entre si, onde destaca-se a eficiência do Exp-6, em segundo lugar o Exp-1 e, por último, o Exp-5. As três retas têm coeficientes de regressão elevados. Isto confirma o fato que seu potencial de resfriamento depende da depressão da temperatura de bulbo úmido. No entanto, o caso dos Exp-4 e Exp-7, que utilizam o ERN (sem EEI), apresentam diferentes inclinações. Estes dois sistemas podem ser classificados em quarto e quinto lugar respectivamente, a partir dos valores de PRM obtidos.

\section{CONCLUSÕES}

A pesquisa analisa as temperaturas características, fatores decrementais e potenciais de resfriamento de cinco sistemas passivos de resfriamento. Os resultados preliminares permitem concluir que: os sistemas avaliados apresentam um potencial médio de resfriamento que varia entre $119 \mathrm{~W} / \mathrm{m}^{2}$-dia e $255 \mathrm{~W} / \mathrm{m}^{2}$-dia, e valores máximos atingindo $319 \mathrm{~W} / \mathrm{m}^{2}$-dia; estas técnicas de resfriamento passivo podem reduzir a temperatura máxima interna em até $10,4^{\circ} \mathrm{C}$ se comparado com a temperatura máxima externa; é possível reduzir a amplitude da temperatura interna em função da amplitude externa (fator decremental) entre 0,36 e 0,48. Estes resultados confirmam a viabilidade de aplicação das técnicas de resfriamento passivo para o condicionamento térmico de edificações no período de verão em Florianópolis. Sendo experimentos com módulos em escala reduzida, os resultados não podem ser extrapolados diretamente para um edifício em escala real. As cargas de calor internas e as diferenças nas propriedades térmicas e ópticas das paredes e no piso do edifício em relação ao ME podem representar uma perda na eficiência da refrigeração em relação ao obtido em experiências, em termos de valores absolutos de temperatura registrados. 


\section{AGRADECIMENTOS}

Os autores gostariam de agradecer à CAPES pelo apoio financeiro concedido ao Prof. Eduardo Manuel González Cruz, dentro do Programa CAPES/Professor visitante do exterior - PVE”, para participar no Programa de Pós-Graduação em Arquitetura e Urbanismo da Universidade Federal de Santa Catarina, durante os anos de 2013 e 2014. Adicionalmente, os autores agradecem ao LEPTEN/CTC/UFSC por facilitar os dados ambientais medidos pela estação BSRN-FLN.

\section{REFERÊNCIAS}

DORNELLES, K.A., RORIZ, M. Thermal inertia, comfort and energy consumption in buildings: a case study in Sao Paulo State-Brasil, International journal for housing science and its applications, Vol. 28 (2004), Part 2, pp. 153-162.

EMPRESA DE PESQUISA ENERGÉTICA (EPE). Balanço Energético Nacional 2013: Ano base 2012. Ministério de Minas e Energia, Brasília - DF - CEP: 70711-902. Rio de Janeiro: EPE, 2013.

GIVONI, B. Experimental studies on radiant and evaporative cooling of roofs. In Proceedings of the International Passive and Hybrid Cooling Conference, Miami Beach, American Section of the International Solar Energy Society, USA, (1981), pp. 279-283.

GIVONI, B. Indoor temperature reduction by passive cooling systems. Solar Energy, Volume 85, Issue 8, August 2011, Pages 1692-1726

GIVONI, B. Passive and Low Energy Cooling of Buildings. Van Nostrand Reinhold. (1994).

GONZALEZ-CRUZ, E. M.; CONZALEZ-GARCIA, S. I. Estudio experimental sobre el comportamiento térmico de un nuevo tipo de techo-estanque para el enfriamiento pasivo en clima húmedo. Ambiente Construído, Porto Alegre, v. 13, n. 4, p. 149-171, out./dez. 2013.

GÜTHS, S., NICOLAU, V. P. Instrumentação em ciências térmicas. Florianópolis, LMPT/UFSC. 1998. 37 p.

KHARRUFA, S.N. Y ADIL, Y. Roof pond cooling of buildings in hot arid climates, Building and Environment, vol. 43 (2008) no. 1, pp. 82-89.

PAPST, A.L. Uso de inércia térmica no clima subtropical. Estudo de caso em Florianópolis-SC. Florianópolis, 1999. 165p. Dissertação (Mestrado em Engenharia Civil) Curso de Pós-Graduação em Engenharia Civil, Universidade Federal de Santa Catarina.

SÁNCHEZ, L. Evaluación de un Techo-estanque Como Sistema de Enfriamiento Pasivo en un Clima Cálido Sub-húmedo. 1993. Tesis de Maestría en Diseño Bioclimático, Universidad de Colima, México.

SZOKOLAY, S.V. Dilemas of warm-humid climate house design: heavy vs. lightweight + cooling effect of air movement, pp. 144-149 in Architecture, city, environment, Proc. PLEA 2000 Conference. Cambridge, UK.

TANG, R., ETZION, Y., ERELL, E. Experimental studies on a novel roof pond configuration for the cooling of buildings. Renewable Energy, Vol. 28 (2003) $\mathrm{N}^{\circ} 10$, pp. 1513-1522. 